\title{
Anisotropic Nanoscale Galvanic Replacement Reactions Studied by Liquid Cell Scanning Transmission Electron Microscopy
}

\author{
Hongyu Sun, Murat Nulati Yesibolati, Silvia A. Canepa, and Kristian Mølhave \\ Department of Micro- and Nanotechnology, Technical University of Denmark, Lyngby, Denmark
}

Anisotropic nanostructures made of noble metals have remarkable electronic, optical, and catalytic properties, giving a broad range of applications in biomedicine, sensors, energy storage and conversion [1]. To tailor these physical/chemical properties and optimize device performance, different structural parameters and chemical composition of the metal nanostructures are tuned by controlling the nucleation and growth during synthesis process, or post treatment methods. Compared to the solid counterparts, hollow or porous anisotropic noble metal nanostructures possess lower mass density and larger specific surface area, which are favorable for reducing the usage of precious metals, increasing contact area with the surrounding reaction medium, accelerating mass and ion transportation, and accommodating local strain and stress in the working conditions [2]. It is therefore of great importance to understand the formation process and synthesize anisotropic noble metal nanostructures with hollow or porous morphology [2].

A galvanic replacement reaction (GRR) is a spontaneous process. One metal (A) is used as a sacrificial template and immersed in a solution containing ions of another metal (B) with higher reduction potential, then metal A will be continuously oxidized and dissolved into the solution, while metal B will be reduced and conformally coated onto the exposed surface of metal A. Recently, GRR has been used to synthesize hollow alloy nanostructures with adjusted composition and morphology [3-5], and Ag nanostructures are widely used as templates due to the facile control of the geometry by colloidal chemistry [5].

By quenching the reaction at a given point in time and characterizing the resulting samples, several possible mechanisms of the hollow nanostructures formation have been proposed. Ex-situ studies provide useful information but direct evidence requires real-time observation of the morphological evolution, and preferably reactions at a single-particle level. In-situ transmission electron microscopy (TEM) combined with a liquid cell has been successfully employed to study the chemical transformations of nanocrystals with subnanometer scale spatial resolution $[6,7]$. In this work, we use in-situ flow LCTEM for real time monitoring of the GRR between different Ag nanostructures, including nanorods (Figure 1), and triangular nanoplates (Figure 2), with gold(III) chloride trihydrate $\left(\mathrm{HAuCl}_{4}\right)$ aqueous solution. Without adding any chelating ligands or scavengers that may modify the reaction process, but by using continuous flow of reagent solution into the liquid cell and controlling the electron flux to a level that does not significantly influence the processes, real time observing and quantitative analysis of the GRR are achieved and compared with the ex-situ control experiments and previous in-situ work [3, 8-10]. Our studies indicate that the GRR process has a high facet selectivity. The detailed reaction progresses are quantitatively analyzed, and compared with the existing models. The present LCTEM results not only provide new insights in the GRR that would be difficult to achieve by other approaches, but also shed light on the synthesis of anisotropic hollow/porous nanostructures with novel properties [11]. 
References:

[1] SE Lohse et al, Chemistry of Materials 26 (2014), p. 34.

[2] X Wang et al, Chemical Reviews 116 (2016), p. 10983.

[3] X Xia et al, Advanced Materials 25 (2013), p. 6313.

[4] E González et al, Science 334 (2011), p. 1377.

[5] L Au et al, Nature Protocols 2 (2007), p. 2182.

[6] FM Ross, Science 350 (2015), p. aaa9886.

[7] SA Canepa et al, The Journal of Physical Chemistry C 122 (2018), p. 2350.

[8]A Courty et al, Nature Communications 5 (2014), p. 4946.

[9] SF Tan et al, ACS Nano 10 (2016), pp. 7689.

[10] SW Chee et al, Nature Communications 8 (2017), p. 1224.

[11] The authors acknowledge funding DTU and from the Danish Research Council for Technology and Production Case No. 12-126194.
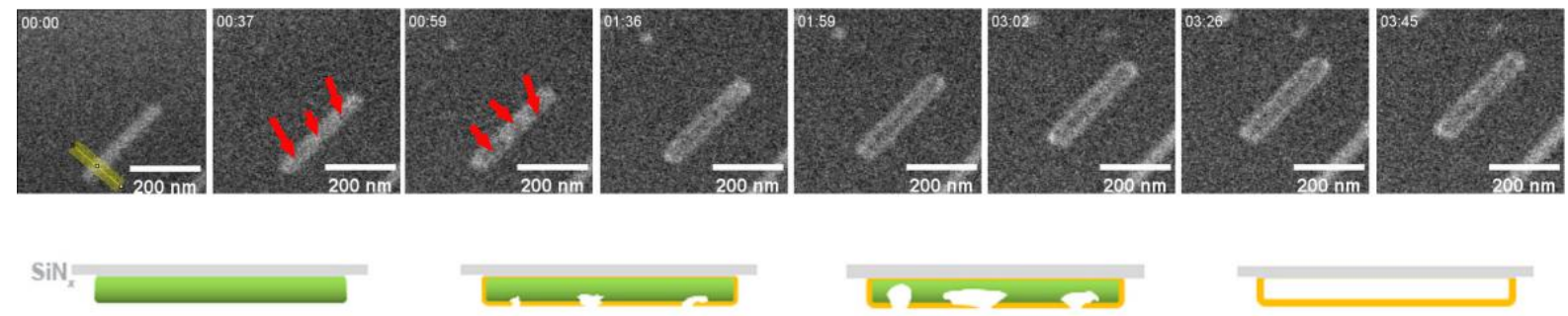

Figure 1. Time series of HAADF-STEM images and schematics illustrating the evolution of a typical $\mathrm{Ag}$ nanorod during the GRR with $\mathrm{HAuCl}_{4}$ solution. The red arrows show the initial pitting positions. Incident electron flux: $119 \mathrm{e}^{-} \mathrm{nm}^{-2} \mathrm{~s}^{-1}$, liquid flow rate: $20 \mu \mathrm{Lmin}^{-1}$.

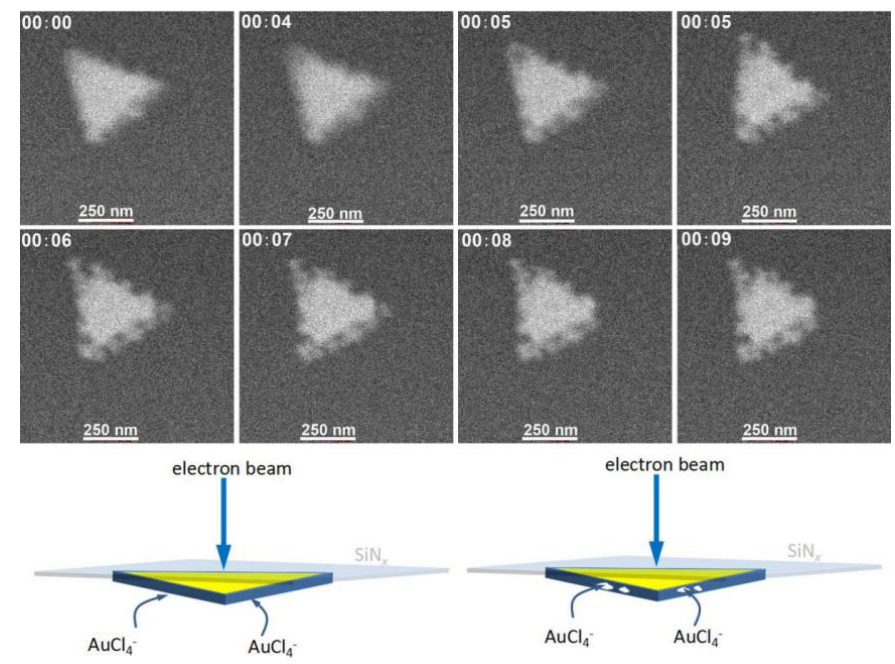

Figure 2. Time series of HAADF-STEM images and schematics showing the morphological change of a triangular Ag nanoplate during the GRR. Incident electron flux: $119 \mathrm{e}^{-} \mathrm{nm}^{-2} \mathrm{~s}^{-1}$, liquid flow rate: 20 $\mu \mathrm{Lmin}^{-1}$. 